\title{
Demonstration of a Squeezed-Light-Enhanced Power- and Signal-Recycled Michelson Interferometer
}

\author{
Henning Vahlbruch, Simon Chelkowski, Boris Hage, Alexander Franzen, Karsten Danzmann, and Roman Schnabel \\ Institut für Atom- und Molekülphysik, Universität Hannover and Max-Planck-Institut für Gravitationsphysik (Albert-Einstein-Institut), \\ Callinstrasse 38, 30167 Hannover, Germany \\ (Received 16 August 2005; published 16 November 2005)
}

\begin{abstract}
We report on the experimental combination of three advanced interferometer techniques for gravitational wave detection, namely, power recycling, detuned signal recycling, and squeezed field injection. For the first time, we experimentally prove the compatibility of especially the latter two. To achieve a broadband nonclassical sensitivity improvement, we applied a filter cavity for compensation of quadrature rotation. The signal-to-noise ratio was improved by up to $2.8 \mathrm{~dB}$ beyond the coherent state's shot noise. The complete setup was stably locked for arbitrary times and characterized by injected single-sideband modulation fields.
\end{abstract}

PACS numbers: 04.80.Nn, 07.60.Ly, 42.50.Dv

Gravitational waves (GWs) have been predicted by Albert Einstein using the theory of general relativity, but so far they have not been observed [1]. An international array of ground-based, kilometer-scale Michelson interferometers has been set up for their first observation, consisting of GEO 600 [2], LIGO [3], TAMA 300 [4], and VIRGO [5]. The goal is to measure a gravitational wave induced strain of space-time of the order of $10^{-21}$ integrated over a bandwidth of a few hundred hertz at acoustic frequencies. Even for kilometer-scale interferometers, the expected signals are so small that several kilowatts of circulating single mode laser radiation are required to push the shot noise below the signal strength. Such high powers cannot be achieved by today's lasers alone. Power recycling [6] is an advanced interferometer technique that aims for increased circulating power. Signal recycling [7] was also invented to improve the signal-to-shot-noise ratio at some detection frequencies. In fact, also the injection of squeezed states was first proposed to reduce shot noise [8]. Later, in the 1980s, it was realized that squeezed states can also be used to reduce the overall quantum noise in interferometers including radiation pressure noise, thereby beating the standard-quantum limit $[9,10]$. Recently, it was discovered that, at radiation pressure dominated frequencies, signal recycling can also be used to beat the standard quantum limit [11]. Gea-Banacloche and Leuchs showed that the techniques of power recycling and squeezed field injection are fully compatible [12]. Chickarmane and Dhurandhar [13] found compatibility of signal recycling and squeezed field injection for the shot-noise limited regime. Furthermore, the analysis by Harms et al. showed that the same is true for detuned signal recycling at shot noise as well as radiation-pressure-noise dominated frequencies [14], thereby proposing that all three techniques can simultaneously be used to reduce quantum noise in interferometers.

All of the GW detectors mentioned above use power recycling; additionally, most of them also use arm cavities for further power buildup. In both cases, additional mirrors form tuned cavities. The GEO 600 detector already successfully uses carrier light detuned signal recycling. Signal recycling is established by an additional mirror placed into the interferometer's dark signal port, forming a signal tuned cavity. This leads to an optical resonance structure in the interferometer's signal transfer function, whose frequency can be changed and matched to an expected signal, for example, emitted by a binary system of two neutron stars or black holes. Signal recycling in combination with power recycling is often called dual recycling and was experimentally demonstrated by Heinzel et al. [15]. Second-generation detectors currently being planned, for example, Advanced LIGO [16], are likely to use this technique. Then, in combination with tuned high finesse arm cavities, the technique is called resonantsideband extraction [17,18]. Squeezed states are envisaged for third-generation detectors, but so far only a few squeezed-light-enhanced interferometers have been demonstrated, e.g., tabletop Mach-Zehnder and polarization interferometers $[19,20]$, respectively. Recently, a squeezing enhanced power-recycled Michelson interferometer has been reported already bearing more resemblance to a GW detector [21].

In this Letter, we report the first power- and signalrecycled Michelson interferometer with broadband sensitivity better than its photon shot noise. Frequency dependent squeezed light generated in an optical parametric amplifier (OPA) in combination with a detuned filter cavity (FC), as proposed in Ref. [22], was injected through the signal-recycling mirror (SRM) into the interferometer's dark port. The whole setup was stably locked in all its degrees of freedom and characterized by a single-sideband modulation field. Our results generally prove the optical compatibility of the three advanced interferometer techniques described above and also demonstrates a readout and control scheme in which no phase modulation control signals contaminate the detection band. 
The main laser source of our experiment was a monolithic nonplanar Nd:YAG ring laser of $2 \mathrm{~W}$ single mode output power at $1064 \mathrm{~nm}$. About $1 \mathrm{~W}$ was used for second harmonic generation (SHG) to produce the necessary pump field for the OPA; see Fig. 1. The residual beam was transmitted through a mode cleaner ring cavity to reduce laser amplitude noise and spatial fluctuations. The outgoing field was used as a local oscillator for the homodyne detector $(50 \mathrm{~mW})$ as well as a seed beam for the OPA $(100 \mathrm{~mW})$, filter cavity locking $(30 \mathrm{~mW})$, and the Michelson $(120 \mathrm{~mW})$. For controlling the OPA cavity length, a Pound-Drever-Hall (PDH) locking scheme with a phase modulation sideband frequency of $153.8 \mathrm{MHz}$ was used. The error signal was fed back to the piezoelectric transducer mounted coupling mirror of the hemilithic cavity. A similar locking technique was used for the SHG. Another locking loop stabilized the phase relation between the fundamental and second harmonic fields inside the OPA. A more detailed description can be found in Ref. [23]. Locking the OPA to deamplification generates a broadband amplitude quadrature squeezed beam of about $92 \mu \mathrm{W}$ at $1064 \mathrm{~nm}$. This beam was then first passed through a Faraday isolator, protecting the OPA from any backscattered light. A $\lambda / 4$ waveplate turned the $s$-polarized beam into a circularly polarized beam which was mode matched into our linear FC. The coupling and end mirrors had reflectivities of $90 \%$ and $99.92 \%$, respectively. The cavity length was electronically stabilized to about $L=1.21 \mathrm{~m}$, resulting in a free spectral range of $124 \mathrm{MHz}$. We applied the PDH locking technique, utilizing a circularly polarized laser beam that carried $134 \mathrm{MHz}$ phase modulation sidebands and was coupled into the filter cavity from the opposite side. Therefore, it was possible to lock the FC stably to a sideband frequency of $\pm 134 \mathrm{MHz}$, which results in a detuning frequency of $\pm 10 \mathrm{MHz}$ due to the free spectral range of $124 \mathrm{MHz}$. This technique avoided unwanted control signals showing up in the detection band. Note that such a signal was present in Figs. 3(a) and 4(a) of Ref. [23] at $15 \mathrm{MHz}$. Locking the filter cavity to either the upper or the lower sideband, the squeezed field was then reflected towards the signal-recycling mirror.

The Michelson interferometer was dual recycled; both recycling cavities had lengths of about $1.21 \mathrm{~m}$ and the reflectivities of the power-recycling mirror (PRM) and the SRM were both $90 \%$; cf. Fig. 1. The interferometer was stabilized on a dark fringe, and the PRM was controlled such that it formed a carrier field resonating cavity together with the two Michelson end mirrors of $99.92 \%$ reflectivity. The finesse of this power-recycling cavity was measured to 60. The signal-recycling cavity (SRC) that was formed by the SRM and the two end mirrors contained no carrier field and could be stably locked to sideband frequencies of $\pm 10 \mathrm{MHz}$. Altogether, three electronic control loops were applied to stabilize the interferometer to this operation point, utilizing two polarization modes and two modulation frequencies. The phase modulations at 15 and $134 \mathrm{MHz}$ were applied to the carrier field before it entered the interferometer, and a $\lambda / 2$ plate in front of the PRM split the incoming beam into $100 \mathrm{~mW}$ of $s$ polarization and $20 \mathrm{MHz}$ of $p$ polarization. The position of the PRM was locked by a PDH technique via the $15 \mathrm{MHz}$ sidebands in the $s$ polarization. Modulation sidebands at $134 \mathrm{MHz}$ in the $s$ and $p$ polarizations were then used to control the dark port and the length of the detuned signalrecycling cavity, respectively. The polarization modes were decoupled by a polarizing beam splitter (PBS) that was placed between the 50/50 beam splitter and the SRM. An arm length difference of the Michelson of $7 \mathrm{~mm}$ was sufficient to provide adequate strong error signals.

The squeezed beam from the detuned locked filter cavity was injected into the SRC, passing a combination of a PBS, a $\lambda / 2$ plate, and a Faraday rotator. This gave spatial

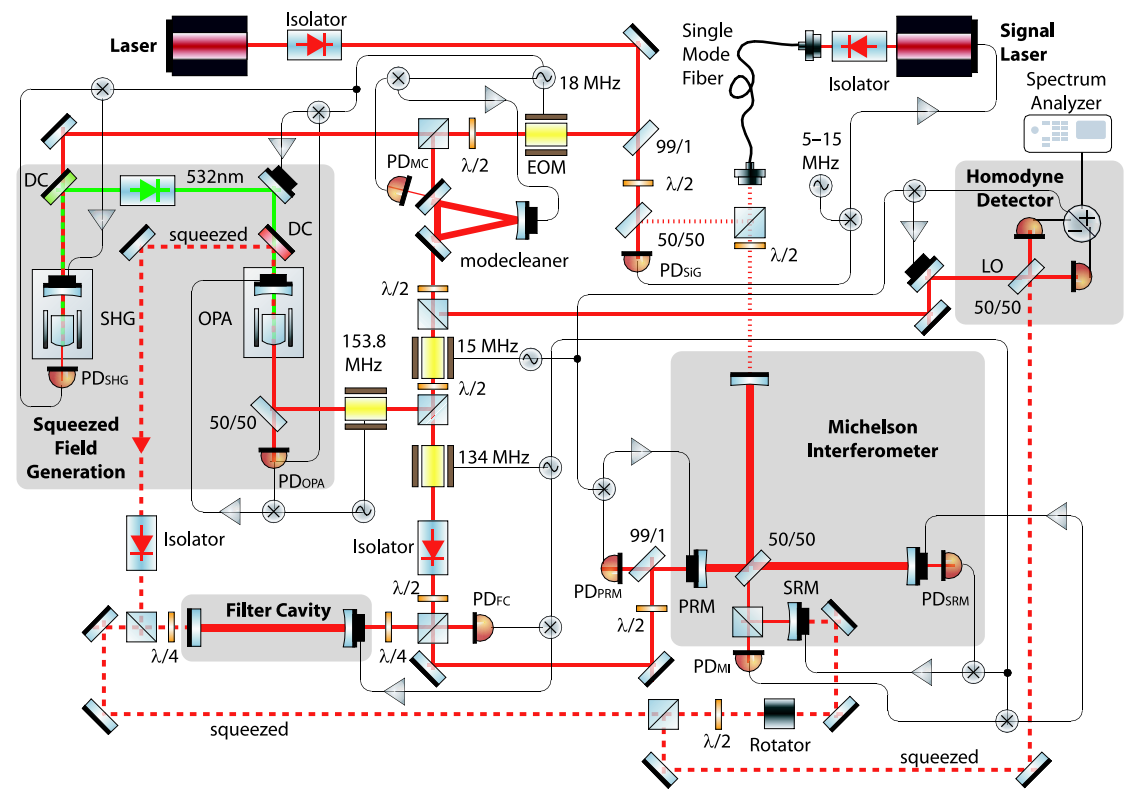

FIG. 1 (color online). Schematic of the experiment. Amplitude squeezed light is generated in an OPA cavity of controlled length. The detuned filter cavity provides frequency dependent squeezing suitable for a broadband quantum noise reduction of a shot-noise limited dualrecycled Michelson interferometer. SHG: second harmonic generation; OPA: optical parametric amplifier; DC: dichroic mirror; LO: local oscillator; PD: photodiode; PRM: powerrecycling mirror; SRM: signal-recycling mirror; $\mathbf{\square}$ : piezoelectric transducer. 
degeneracy between the reflected squeezing and the signal output beam of the interferometer. The combined field was guided to a homodyne detector that was built from two electronically and optically matched photodetectors based on Epitaxx ETX1000 photodiodes. All spectra presented in this paper were analyzed with $100 \mathrm{kHz}$ resolution bandwidth and $100 \mathrm{~Hz}$ video bandwidth, averaging over 5 subsequent measurements.

The setup described so far facilitates the measurement of the interferometer's noise transfer function. The measurement of the signal transfer function and, therefore, the combined signal-to-noise ratio required the generation of a modulation signal of known strength and tunable sideband frequency. We chose to inject a single-sideband modulation field into the interferometer, similar in design to Ref. [24]. Such a field is generically different from a phase modulation, for example, generated by a gravitational wave. However, it has been shown in Ref. [25] that a shot-noise limited signal-recycled interferometer with detuning larger than the SRC bandwidth cannot be significantly improved by frequency dependent homodyning, socalled variational output. The reason is that only a single sideband is supported and injecting a single-sideband modulation is therefore a meaningful method to characterize the signal-recycled interferometer. The signal was generated utilizing a second monolithic nonplanar Nd:YAG ring laser. This laser was frequency locked to the main laser by a phase lock loop, with tunable beat frequency in the range of 5-15 MHz between both light sources. This signal laser beam was injected through one of the interferometer end mirrors as shown in Fig. 1.

Figure 2 shows the squeezed-light-enhanced quantum noise performance of our dual-recycled interferometer. By using frequency dependent squeezed light, the quantum noise can be reduced below the shot-noise level (a) for all sideband frequencies shown (d). If, instead, no filter cavity is used, the quantum noise is increased at some sideband

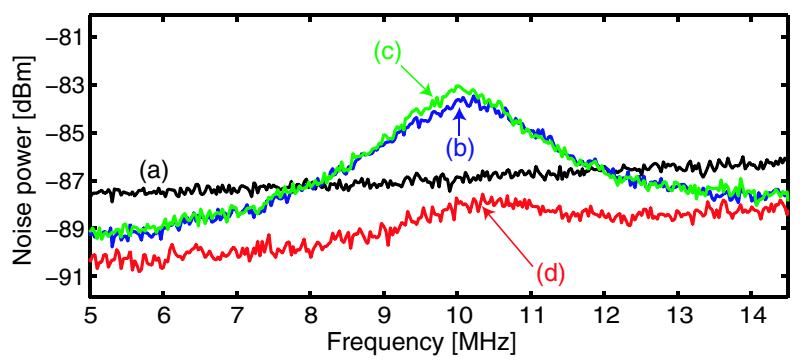

FIG. 2 (color online). Amplitude quadrature noise power spectra from homodyne detection: (a) shot noise, (b) frequency dependent squeezing after reflection at the $+10 \mathrm{MHz}$ detuned signal-recycling cavity (SRC) without reflection from the detuned FC, (c) frequency dependent squeezing after reflection at the $-10 \mathrm{MHz}$ detuned filter cavity (FC) without reflection from the detuned SRC, (d) regained broadband squeezed-lightenhanced performance achieved by reflecting the squeezed light from both cavities with opposite detunings. frequencies (b). This effect is a consequence of a rotation of the field's quadratures when reflected from a detuned cavity [22]. While the OPA generated an amplitude squeezed beam, the squeezing ellipse is rotated such that antisqueezing is detected around the SRC-detuning frequency by the homodyne detector. This effect has first been observed and characterized in Ref. [23]. The filter cavity compensates this effect while being locked to the same detuning frequency but with opposite sign. The remaining hump around $10 \mathrm{MHz}$ in curve (d) arises from additional losses inside the SRC caused mainly by the PBS. For this reason, the squeezing got reduced most exactly at the detuning frequency.

The mode matching efficiencies of the squeezed field into the FC and SRC were 95\% in both cases. Additional losses on the squeezing arose from the OPA escape efficiency of $90 \%$, transmittance of the isolator including double passing the Faraday rotator of $91 \%$, mode matching efficiency at the homodyne detector of $95 \%$, and quantum efficiency of photodiodes of $93 \%$, adding up to an overall efficiency of $65 \%$. The result was a detected nonclassical noise suppression of $2.8 \mathrm{~dB}$ at $5 \mathrm{MHz}$. The poorer squeezing of about $2.0 \mathrm{~dB}$ at $14 \mathrm{MHz}$ was due to the limited bandwidth of the OPA of $20 \mathrm{MHz}$. In principle, the bandwidth can be increased by higher coupler transmission, which in turn requires a higher pump intensity, but since, in GW detectors, squeezing at acoustic frequencies will apply, the OPA bandwidth limitation will not be an issue. We note that all measured spectra were at least $5 \mathrm{~dB}$ above the detection dark noise, which was taken into account.

Figure 3 demonstrates the squeezing enhanced signalto-noise ratio (SNR) of single-sideband signals. The Michelson interferometer was stably locked with a $+10 \mathrm{MHz}$ detuned signal-recycling cavity, while a single sideband was subsequently injected at ten different sideband frequencies, and the spectra were measured with and without frequency dependent squeezed field injection. The experimental results show an increase of the SNR over the whole detection bandwidth. In comparison with the shotnoise limited signals (c) the SNR was improved by up to $2.8 \mathrm{~dB}$ into the nonclassical regime (b).

In conclusion, we have experimentally demonstrated the compatibility of power recycling, carrier detuned signal recycling, and squeezed field injection. Broadband nonclassical noise suppression was achieved by employing a detuned filter cavity for compensation of quadrature rotation. The optical layout of our demonstration experiment can directly be applied to improve the sensitivity of large scale signal-recycled interferometers at their shot-noise limited detection frequencies, typically above $1 \mathrm{kHz}$. The scheme demonstrated also directly applies to the shot-noise limited resonant-sideband extraction topology which is planned for the Advanced LIGO detector (LIGO II) [16]. Then the filter cavity needs to be adapted to compensate for the quadrature rotation due to the resonant-sideband extraction cavity [14]. 


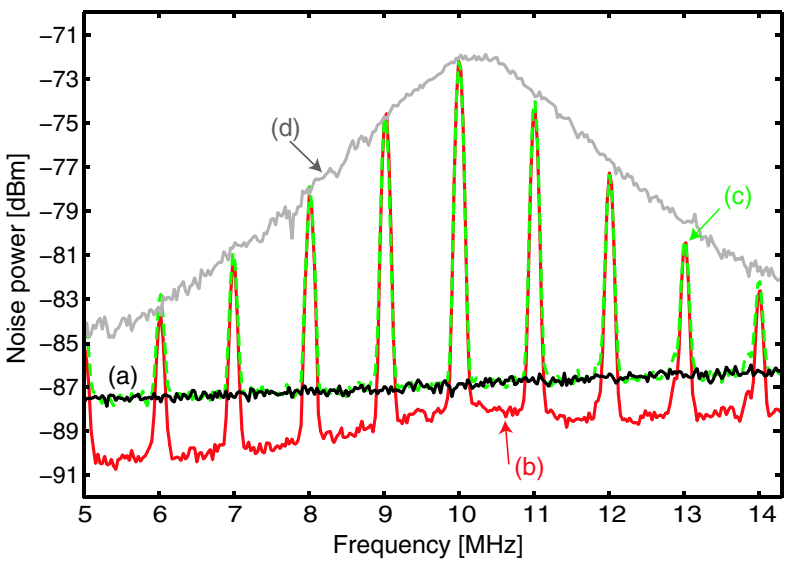

FIG. 3 (color online). Amplitude quadrature power spectra of the dual-recycled Michelson interferometer with and without nonclassical noise reduction. A single-sideband modulation was injected at certain frequencies to characterize the signalto-noise ratio. (a) Shot noise measured with a blocked signal beam at the homodyne detector; (b) (dashed line) shot-noise limited signals; (c) broadband squeezing enhanced signal-tonoise ratios of up to $2.8 \mathrm{~dB}$ below shot noise. Utilizing the max hold function of the spectrum analyzer, the optical transfer function of the SRC was mapped out by continuously sweeping the injected signal (d).

After applying classical noise suppression to enable squeezing in the GW band as demonstrated in Ref. [26], our squeezed light source would be directly applicable to all current and probably next-generation detectors since the same laser wavelength is used. Future detectors are also expected to be quantum noise limited at lower frequencies due to backaction noise (radiation-pressure noise). Then the demonstrated experiment can also provide a broadband nonclassical sensitivity improvement if additional filter cavities are used. Then if the initial power- and signalrecycled interferometer is already at the standard-quantum limit, squeezed field injection can provide quantum nondemolition performance [14,22,27]. In our experiment, we chose a homodyne readout with external local oscillator. This is in accordance with theoretical investigations that considered a readout scheme that can switch between arbitrary quadrature angles $[11,14]$. Such a local oscillator might be generated in a large scale GW detector from an unused reflection from the beam splitter antireflection coating. We believe that a realistic goal for future GW detectors is a $6 \mathrm{~dB}$ improvement of power noise spectral density. This requires the generation of a $10 \mathrm{~dB}$ squeezed field at detection frequencies and an overall loss of less than $17 \%$. A detailed estimation of expected individual loss contributions in squeezing enhanced GW detectors will be presented elsewhere [28].

This work has been supported by the Deutsche Forschungsgemeinschaft and is part of Sonderforschungsbereich 407.
[1] K.S. Thorne, in 300 Years of Gravitation, edited by S.W. Hawking and W. Isreal (Cambridge University Press, Cambridge, England, 1987), pp. 330-458.

[2] B. Willke et al., Classical Quantum Gravity 19, 1377 (2002).

[3] A. Abramovici et al., Science 256, 325 (1992).

[4] M. Ando et al., Phys. Rev. Lett. 86, 3950 (2001).

[5] F. Acernese et al., Classical Quantum Gravity 21, S709 (2004).

[6] R. W.P. Drever et al., in Quantum Optics, Experimental Gravitation, and Measurement Theory, edited by P. Meystre and M. O. Scully (Plenum, New York, 1983), pp. 503514.

[7] B. J. Meers, Phys. Rev. D 38, 2317 (1988).

[8] C. M. Caves, Phys. Rev. D 23, 1693 (1981).

[9] W.G. Unruh, in Quantum Optics, Experimental Gravitation, and Measurement Theory, edited by P. Meystre and M. O. Scully (Plenum, New York, 1983), pp. 647-660.

[10] M. T. Jaekel and S. Reynaud, Europhys. Lett. 13, 301 (1990).

[11] A. Buonanno and Y. Chen, Classical Quantum Gravity 18, L95 (2001).

[12] J. Gea-Banacloche and G. Leuchs, J. Mod. Opt. 34, 793 (1987).

[13] V. Chickarmane and S. V. Dhurandhar, Phys. Rev. A 54, 786 (1996).

[14] J. Harms, Y. Chen, S. Chelkowski, A. Franzen, H. Vahlbruch, K. Danzmann, and R. Schnabel, Phys. Rev. D 68 , 042001 (2003).

[15] G. Heinzel, K.A. Strain, J. Mizuno, K. D. Skeldon, B. Willke, W. Winkler, R. Schilling, A. Rüdiger, and K. Danzmann, Phys. Rev. Lett. 81, 5493 (1998).

[16] E. Gustafson, D. Shoemaker, K. Strain, and R. Weiss, LSC White Paper on Detector Research and Development, LIGO Document No. T990080-00-D, Caltech/MIT, 1999. See also www.ligo.caltech.edu/.

[17] J. Mizuno, K. A. Strain, P. G. Nelson, J. M. Chen, R. Schilling, A. Rüdiger, W. Winkler, and K. Danzmann, Phys. Lett. A 175, 273 (1993).

[18] K. Strain et al., Appl. Opt. 42, 1244 (2003).

[19] M. Xiao, L.-A. Wu, and H. J. Kimble, Phys. Rev. Lett. 59, 278 (1987).

[20] P. Grangier, R. E. Slusher, B. Yurke, and A. LaPorta, Phys. Rev. Lett. 59, 2153 (1987).

[21] K. McKenzie, D. A. Shaddock, D. E. McClelland, B. C. Buchler, and P. K. Lam, Phys. Rev. Lett. 88, 231102 (2002).

[22] H. J. Kimble, Y. Levin, A. B. Matsko, K. S. Thorne, and S. P. Vyatchanin, Phys. Rev. D 65, 022002 (2002).

[23] S.Chelkowski, H. Vahlbruch, B. Hage, A. Franzen, N. Lastzka, K. Danzmann, and R. Schnabel, Phys. Rev. A 71, 013806 (2005).

[24] G. de Vine, D. A. Shaddock, and D.E. McClelland, Classical Quantum Gravity 19, 1561 (2002).

[25] R. Schnabel, J. Harms, K. A. Strain, and K. Danzmann, Classical Quantum Gravity 21, S1045 (2004).

[26] K. McKenzie, N. Grosse, W. P. Bowen, S. E. Whitcomb, M. B. Gray, D. E. McClelland, and P. K. Lam, Phys. Rev. Lett. 93, 161105 (2004).

[27] A. Buonanno and Y. Chen, Phys. Rev. D 69, 102004 (2004).

[28] H. Vahlbruch et al. (to be published). 